Pacific Journal of Mathematic 


\title{
ON A THEOREM OF BEURLING AND KAPLANSKY
}

\author{
M. Cotla R
}

1. Introduction. The object of this paper is to remark that a natural and simple proof of the theorem of Beurling and Kaplansky (Theorem 1 below) can be obtained by adapting to general groups a classical proof already given in the books of Wiener [8] and Zygmund [9]. In fact, Theorem 1 is an immediate consequence of a lemma (Lemma 1 below) which was proved by these authors in the case when the group is the integers or the real numbers. An easy generalization of Lemma 1 (Lemma 2 below) yields immediately the generalization of the Beurling and Kaplansky theorem stated as Theorem 2 below. For the history of the development of this theorem, see [3, p. 149] and [5]; the book [3] did not appear until the present paper had been submitted, but it seemed wise to add the reference.

2. Statement of results. Let $A=\{a, b, \ldots\}$ be a locally compact abelian group and $X=\{x, y, \cdots\}$ the dual group (the group operations will be written multiplicatively ). Let

$$
L^{1}(A)=\{f, g, h, p, \cdots\}
$$

denote the set of all integrable functions with respect to the Haar measure of $A$,

$$
\|f\|=\|f\|_{1}
$$

the $L^{1}$-norm of $f, \hat{f}(x)$ the Fourier transform of $f(a)$,

$$
f_{1} * f_{2}
$$

the product of convolution (that is, the product in the group algebra),

$$
f_{1} f_{2}=f_{1}(a) f_{2}(a)
$$

the ordinary product of functions, and

$$
(x, a)=x(a)=a(x)
$$

Received January 12, 1953. The author is a fellow of the John Simon Guggenheim Memorial Foundation.

Pacific J. Math. 4 (1954), 459-465 
the value of the character $x \in X$ at the point $a \in A$. Subsets of $A$ will be denoted by $C, D, \ldots$, subsets of $X$ by $P, Q, S, \ldots$, and subsets of $L^{1}(A)$ by $I, J, \ldots$.

The spectrum $S(f)$ of a function $f \in L^{1}(A)$ is the set of the points $x \in X$ such that $\hat{f}(x)=0$, and the spectrum $S(I)$ of a set $I \subset L^{1}(A)$ is the set of the points $x \in X$ such that $\hat{f}(x)=0$ for all $f \in I$.

We suppose known the following Tauberian theorem of Segal and Godement ( see $[1]$ or $[4])$.

THEOREM A. If $I$ is a closed ideal of $L^{1}(A)$, and $f \in L^{1}(A)$ is such that $S(I)$ is interior to $S(f)$, then $f \in I$.

Theorem $\mathrm{A}$ is a consequence of the regularity (in the sense of Silov) of the algebra $L^{1}(A)$, and the following Lemma A (see [7], [1], or [4]).

Lemma A. Given $f \in L^{1}(A)$ and $\epsilon>0$, there is a function $g \in L^{1}(A)$ with the following properties:

(i) $\hat{f}(x)=0$ implies $\hat{g}(x)=0$; that is, $S(f) \subset S(g)$.

(ii) If $h=f-g$, then $\hat{h}(x)$ vanishes in a neighborhood of the point $\infty$ (that is outside of a compact set $P \subset X)$.

(iii) $\|g\| \leq \epsilon$.

It is known [6] that Theorem A is not true if $S(f)$ is merely contained in but not interior to $S(f)$; however, if $S(I)$ consists of a single point, the following theorem is true:

THEOREM 1 (Beurling and Kaplansky). If $I$ is a closed ideal such that $S(I)$ consists of a single point $x_{0}$, then $S(f) \supset S(I)$ implies $f \in I$.

This is a special case of the following:

THEOREM 2. Let I be a closed ideal such that the boundary $P$ of $S(I)$ is a reducible set (or that the intersection of $P$ with the boundary of $S(f)$ is a reducible set). Then $S(f) \supset S(I)$ implies $f \in I$.

A set is said to be reducible if it contains no nonvoid perfect subsets.

Theorem 1 was proved by Peurling in the case when $A$ consists of the real numbers, using complex-variable methods. Kaplansky proved the theorem in the general case using the structure theory of groups. A direct and simple proof of Theorem 1 is given in a recent paper of Helson [2], and in the same paper is given a complete proof of Theorem 2 . 
We want to show that a still more natural and simple proof of Theorems 1 and 2 can be obtained as follows.

2. Proofs. We first reduce Theorem 1 to the following Lemma 1 (observe that Lemma $\mathrm{A}$ is obtained from Lemma 1 by replacing the point $x_{0}$ by $\infty$ ).

LEMMA 1. Given a point $x_{0} \in S(f), f \in L^{1}(A)$, and $\epsilon>0$, there is a function $g \in L^{1}(A)$ with the following properties:

(i) $S(f) \subset S(g)$ :

(ii) if $h=f-g$, then $\hat{h}(x)$ vanishes in a neighborhood $U\left(x_{0}\right)$ of the point $x_{0}$;

(iii) $\|g\| \leq \epsilon$.

It is easy to see that Theorem 1 is an immediate consequence of Lemma 1 and Theorem A. In fact, if $S(I)$ consists of a single point $x_{0} \in S(f)$, then by Lemma 1 there is a function $h$ such that $\|f-h\|<\epsilon$, and $x_{0}$ is interior to $S(h)$; hence, by Theorem $\mathrm{A}, h \in I$. Since $\epsilon$ is arbitrary and $\|f-h\| \leq \epsilon$, it follows that $f \in I$, and this proves Theorem 1 .

Similarly it is easy to see that Theorem 2 is an immediate consequence of Theorem A, Lemma A, and the following Lemma 2.

LEMMA 2. Given a compact reducible set $Q \subset S(f), f \in L^{1}(A)$, and $\epsilon>0$, there is a function $g \in L^{1}(A)$ with the following properties:

(i) $S(f) \subset S(g)$;

(ii) if $h=f-g$, then $\hat{h}(x)$ vanishes in a neighborhood $U(Q)$ of the set $Q$;

(iii) $\|g\| \leq \epsilon$.

Hence Theorems 1 and 2 will be proved if we prove Lemmas 1 and 2 .

3. Proof of Lemma 1. Without loss of generality we may suppose $x_{0}=1=$ unit of $X$. Then by hypothesis

$$
\hat{f}\left(x_{0}\right)=\int_{A} f(a) d a=0 .
$$

Given $\epsilon>0$, there is a compact set $C \subset A$ such that

$$
\int_{A-C}|f(a)| d a<\epsilon / 4,
$$


hence also

$$
\left|\int_{C} f(a) d a\right|=\left|\int_{A-C} f(a) d a\right|<\epsilon / 4
$$

If $p(a)$ is any function from $L^{1}(A)$, and $g=p * f$, we have

$$
g(a)=\int_{A} f(b) p\left(a b^{-1}\right) d b=\int_{C}+\int_{A=C} f(b) p\left(a b^{-1}\right) d b,
$$

(3)

$$
\begin{aligned}
&\|g\| \leq \int_{A}\left|\int_{C} f(b) p\left(a b^{-1}\right) d b\right| d a \\
& \quad+\int_{A}\left|\int_{A-C} f(b) p\left(a b^{-1}\right) d b\right| d a=M+N .
\end{aligned}
$$

Using (1) and (2), and denoting the characteristic function of the set $C^{\prime}=A-C$ by $\phi_{C^{\prime}}$, we have

$$
\begin{aligned}
N & =\int_{A}\left|\int_{A} f(b) \phi_{C^{\prime}}(b) p\left(a b^{-1}\right) d b\right| d a \\
& =\left\|\left(f \phi_{C^{\prime}}\right) * p\right\| \leq\left\|f \phi_{C^{\prime}}\right\| \cdot\|p\| \\
& =\|p\| \cdot \int_{C^{\prime}}|f(a)| d a \leq \epsilon / 4 \cdot\|p\|,
\end{aligned}
$$

$$
\begin{aligned}
M \leq & \int_{A}\left|\int_{C} f(b)\left[p\left(a b^{-1}\right)-p(a)\right] d b\right| d a \\
& +\int_{A}\left|\int_{C} f(b) d b\right||p(a)| d a \\
\leq & \left.\sup _{b \in C} \int_{A}\left|p\left(a b^{-1}\right)-p(a)\right| d a\right\}\|f\|+\epsilon / 4\|p\| .
\end{aligned}
$$

Let us denote $p\left(a b^{-1}\right)$ by $p^{b}(a)$; then

$$
\|g\| \leq \epsilon / 2\|p\|+\|f\| \sup _{b \in C}\left\|p^{b}-p\right\| .
$$




$$
\hat{g}(x)=\hat{f}(x) \hat{p}(x),
$$

$\hat{f}(x)=0$ implies $\hat{g}(x)=0$, and inequality (4) shows that Lemma 1 will be proved if we prove the following proposition.

Proposition A. Given $\epsilon>0$ and a compact set $C \subset A$, there is a function $p(a)$ such that:

a) $p \in L^{1}(A)$ and $\|p\| \leq 2$;

b) there is a neighborhood $U(1)$ of the point $1 \in X$ such that $\hat{p}(x)=1$ for $x \in U(1)$;

e) $\left\|p^{b}-p\right\|<\epsilon$ for $b$ in the compact set $C$.

Proof of Proposition A. Take two compact neighborhoods $V$ and $V^{\prime}$ of the $1 \in X$, of measures $\eta$ and $\eta^{\prime}$, and such that

$$
\bar{V} \subset V^{\prime} ; \eta^{\prime} \leq 4 \eta
$$

and define

$$
\hat{\dot{p}}(x)=1 / \eta\left\{\hat{\phi}_{V} * \hat{\phi}_{V},\right\}=1 / \eta\left\{\hat{\phi} * \hat{\phi}^{\prime}\right\},
$$

where $\hat{\phi}=\hat{\phi}_{V}\left(\hat{\phi}^{\prime}=\hat{\phi}_{V^{\prime}}\right)$ is the characteristic tunction of the set $V\left(V^{\prime}\right)$. Since $\hat{\phi}, \hat{\phi}^{\prime} \in L^{2}(X)$, by Plancherel's theorem $\hat{p}(x)$ is the Fourier transform of a function $p(a) \in L^{1}(A)$. Since $\bar{V} \subset V^{\prime}$, there is a neighborhood $U=U(1)$ such that $V \cdot U \subset V^{\prime}$, and from (6) it is clear that $\hat{p}(x)=1$ for $x \in U$. Using the Plancherel theorem it is easy to see that $p(a)$ satisfies also the conditions a) and c), provided $V^{\prime}$ is taken small enough (cfr. [5]). For instance, let us prove condition $\mathrm{c})$. Since the Fourier transform of $\phi^{b}-\phi$ is $\hat{\phi}(x)[(x, b)-1]$, and since $\hat{\phi}(x)=0$ outside of $V^{\prime} \cdot V^{\prime}$, it follows that if $b \in C$, and $V^{\prime}$ is small enough, then

$$
\left\|\phi^{b}-\phi\right\|_{2}=\|[(x, b)-1] \hat{\phi}\|_{2} \leq \epsilon_{1}\|\hat{\phi}\|_{2}=\epsilon_{1} \eta^{1 / 2}
$$

for every $b \in C$, where $\epsilon_{1}>0$ is arbitrarily small. Since

$$
p(a)=\phi(a) \phi^{\prime}(a) / \eta,
$$

by Plancherel's theorem,

$\left\|p^{b}-p\right\|_{1}=1 / \eta\left\|\phi \phi^{\prime}-\phi^{b} \phi^{\prime b}\right\| \leq 1 / \eta\left[\left\|\phi^{\prime}\left(\phi-\phi^{b}\right)\right\|+\left\|\phi^{b}\left(\phi^{\prime}-\phi^{\prime b}\right)\right\|\right]$ 


$$
\leq 1 / \eta\left[\left\|\phi^{\prime}\right\|_{2} \epsilon_{1}\|\phi\|_{2}+\|\phi\|_{2} \epsilon_{1}\left\|\dot{\phi}^{\prime}\right\|_{2}\right] \leq 2 \epsilon_{1}\left(\eta \eta^{\prime}\right)^{1 / 2} / \eta \leq 4 \epsilon_{1},
$$

and this proves condition c).

REMARK. As we already mentioned, the foregoing proof of Lemma 1 is an adaptation of a proof given in Zygmund's book. Zygmund considers the particular case when $A$ consists of the integers and $X$ is the unit circle, so that the functions $\hat{f}(x)$ are periodic functions with absolutely convergent Fourier series, and he takes for $\hat{p}(x)$ the function

$$
\begin{aligned}
& \hat{p}(x)=1 \text { if }|x| \leq \eta, \\
& \hat{p}(x)=0 \text { if }|x| \geq 2 \eta, \\
& \hat{p}(x) \text { linear if } \eta \leq|x| \leq 2 \eta .
\end{aligned}
$$

Then he proves that the total variation of the derivative of the function is bounded by a fixed number, and from this he deduces properties a), b), c) of the function $p(a)$. This is the only point in Zygmund's proof which does not apply to general groups; however, it is easy to see that the function $\hat{p}$ used by Zygmund is exactly what formula (6) reduces to when $V$ is taken to be an interval, and thus the proof can be adapted to the general case.

4. Proof of Lemma 2. Let $Q \subset S(f)$ be a compact reducible set, and let $Q^{(1)}=Q^{\prime}$ be the set of the points $x$ such that any neighborhood of $x$ contains an infinite subset of $Q$. Define

$$
Q^{(2)}=\left(Q^{(1)}\right)^{\prime},
$$

and form in the usual way the sequence of derivative sets:

$$
Q \supset Q^{(1)} \supset Q^{(2)} \supset \cdots \supset Q^{(\alpha)} \supset \cdots
$$

Let $w$ be such that

$$
Q^{(w)}=Q^{(w+1)}
$$

then $Q^{(w)}$ is a perfect set; and since $Q$ is reducible, $Q^{(w)}=0$. If $w=1$, then $Q$ is a finite set and $n$ successive applications of Lemma 1 yields Lemma 2 in this case. We will now prove Lemma 2 by induction on $w$.

Suppose that Lemma 2 is true if $Q^{(w)}=0$ for $w<w_{0}$; we shall prove that 
it is also true if $Q^{(u)}=0$ for $w=w_{0}$. Consider first the case when $w_{0}=w^{\prime}+1$. Then $Q^{\left(w^{\prime}\right)}$ is a finite set, and hence there is a function $h \in L^{1}(A)$ such that

$$
\|f-h\| \leq \epsilon / 2, S(f) \subset S(h),
$$

and $\hat{h}(x)$ vanishes on an open set $U \supset Q^{\left(w^{\prime}\right)}$. Since $Q-U$ has the property

$$
(Q-U)^{\left(w^{\prime}\right)}=0
$$

and $w^{\prime}<w_{0}$, by the inductive assumption there is a function $h^{\prime}$ such that

$$
S(f) \subset S(h) \subset S\left(h^{\prime}\right),\left\|h-h^{\prime}\right\| \leq \epsilon / 2,
$$

and $\hat{h}^{\prime}(x)$ vanishes on an open set $U^{\prime} \supset Q-U$. Hence $\hat{h}^{\prime}(x)$ vanishes on $U \cup U^{\prime} \supset$ $Q$, and

$$
\left\|f-h^{\prime}\right\| \leq\|f-h\|+\left\|h-h^{\prime}\right\| \leq 2 \epsilon / 2=\epsilon \text {. }
$$

If $w_{0}$ is not of the form $w^{\prime}+1$, then by definition

$$
Q^{\left(w_{0}\right)}=\bigcap_{w<w_{0}} Q^{(w)}
$$

hence for some $w^{\prime}<w_{0}$ we must have $Q^{\left(w^{\prime}\right)}=0$, and by the inductive assumption Lemma 2 is true in this case.

This proves Lemma 2.

\section{REFERENCES}

1. R. Godement, Théorems tauberien et théorie spectral, Ann. Sci. Ecole Norm. Sup. (3) 64 (1947), 119-138.

2. H. Helson, Spectral synthesis of bounded functions, Ark. N!at. 1 (1952), 497-502.

3. L. H. Loomis, An introduction to abstract harmonic analysis, New York, 1953.

4. H. Mirkil, The work of Silov on B-algebras, Univ. of Chicago, 1952.

5. H. Reiter, Investigations in harmonic analysis, Trans Am. Math. Soc. 73 (1952), $401-427$.

6. L. Schwartz, Sur une propriété de synthèse spectrale dans les groupes non compacts, C. R. Acad. Sci. Paris, 227 (1948), 424-426.

7. G. Silov, On regular normed rings, Trav. Inst. Math. Stekloff 21 (1947), 118 pp.

8. N. Wiener, The Fourier integral and its applications, Cambridge, 1932.

9. A. Zygmund, Trigonometrical series, Warsaw, 1935. 



\section{PACIFIC JOURNAL OF MATHEMATICS}

\section{EDITORS}

\author{
M.M. SCHIFFE R* \\ Stanford University \\ Stanford, California \\ E. HEWITT \\ University of Washington \\ Seattle 5, Washington
}

R.P. DILWORTH

California Institute of Technology Pasadena 4, California

E.F. BECKENBACH**

University of California

Los Angeles 24, California

\section{ASSOCIATE EDITORS}

$\begin{array}{llll}\text { H. BUSEMANN } & \text { P.R. HALMOS } & \text { BORGE JESSEN } & \text { J. J. STOKER } \\ \text { HERBERT FEDERER } & \text { HEINZ HOPF } & \text { PAUL LÉVY } & \text { E.G. STRAUS } \\ \text { MARSHALL HALL } & \text { R.D. JAMES } & \text { GEORGE PÓLYA } & \text { KÔSAKU YOSIDA }\end{array}$

\section{SPONSORS}

UNIVERSITY OF BRITISH COLUMBIA CALIFORNIA INSTITUTE OF TECHNOLOGY UNIVERSITY OF CALIFORNIA, BERKELEY UNIVERSITY OF CALIFORNIA, DAVIS UNIVERSITY OF CALIFORNIA, LOS ANGELES UNIVERSITY OF CALIFORNIA, SANTA BARBARA UNIVERSITY OF NEVADA OREGON STATE COLLEGE UNIVERSITY OF OREGON
UNIVERSITY OF SOUTHERN CALIFORNIA STANFORD RESEARCH INSTITUTE STANFORD UNIVERSITY WASHINGTON STATE COLLEGE UNIVERSITY OF WASHINGTON

AMERICAN MATHEMATICAL SOCIETY HUGHES AIRCRAFT COMPANY

Mathematical papers intended for publication in the Pacific Journal of Mathematics should be typewritten (double spaced), and the author should keep a complete copy. Manuscripts may be sent to any of the editors. Manuscripts intended for the outgoing editors should be sent to their successors. All other communications to the editors should be addressed to the managing editor, E.G. Straus, at the University of California Los Angeles 24, California.

50 reprints of each article are furnished free of charge; additional copies may be obtained at cost in multiples of 50 .

The Pacific Journal of Mathematics is published quarterly, in March, June, September, and December. The price per volume (4 numbers) is $\$ 12.00$; single issues, $\$ 3.50$; back numbers (Volumes $1,2,3$ ) are available at $\$ 2.50$ per copy. Special price to individual faculty members of supporting institutions and to individual members of the American Mathematical Society: $\$ 4.00$ per volume; single issues, $\$ 1.25$.

Subscriptions, orders for back numbers, and changes of address should be sent to the publishers, University of California Press, Berkeley 4, California.

Printed at Ann Arbor, Michigan. Entered as second class matter at the Post Office, Berkeley, California.

* To be succeeded in 1955, by H.L. Royden, Stanford University, Stanford, California.

** To be succeeded in 1955, by E.G. Straus, University of California, Los Angeles 24, Calif.

UNIVERSITY OF CALIFORNIA PRESS - BERKELEY AND LOS ANGELES 


\section{Pacific Journal of Mathematics \\ Vol. 4, No. $3 \quad$ July, 1954}

Nelson Dunford, Spectral operators ........................ 321

John Wermer, Commuting spectral measures on Hilbert space.......... 355

Shizuo Kakutani, An example concerning uniform boundedness of spectral measures...................................... 363

William George Bade, Unbounded spectral operators .............. 373

William George Bade, Weak and strong limits of spectral operators ...... 393

Jacob T. Schwartz, Perturbations of spectral operators, and applications. I. Bounded perturbations ........................... 415

Mischa Cotlar, On a theorem of Beurling and Kaplansky............... 459

George E. Forsythe, Asymptotic lower bounds for the frequencies of certain polygonal membranes ............................... 467 